\title{
Rabbit Plasmacytoma
}

National Cancer Institute

\section{Source}

National Cancer Institute. Rabbit Plasmacytoma. NCI Thesaurus. Code C133738.

Plasmacytoma that occurs in a rabbit. 